\title{
A NEW FLOW
}

\author{
BY DENNIS SULLIVAN
}

Communicated December 8, 1975

THEOREM (a) On the compact five manifold $S^{3} \times S^{1} \times S^{1}$ there is a nondegenerate flow with every orbit periodic but with lowest periods unbounded.

(b) Each four manifold $S^{\mathbf{3}} \times S^{\mathbf{1}} \times\{\theta\}$ is fibred in circles by the flow. The base or quotient is $S^{2} \times S^{1}$ for $\theta=0$ (near which the periods are unbounded) and $S^{3}$ for $\theta \neq 0$ (where the periods are continuous).

Proof . (i) Using (over and over) the deformation of the equator of $S^{2}$ which introduces two coils, we construct a one parameter family of immersed curves on $S^{2},\left\{\gamma_{t}, 0<t \leqslant \pi\right\}$, so that the geodesic curvature of each point on $\gamma_{t}$ goes uniformly to infinity as $t$ approaches zero.

(ii) Lift $\gamma_{t}$ to the unit tangent bundle of $S^{2}$ and then to $S^{3}$ by the double covering.

(iii) Fill up $S^{3} \times S^{1} \times\{\theta\}$ by the $S^{3}$-translates of the graph of this lift for $0 \neq|\theta|=t$, where $-\pi \leqslant \theta \leqslant \pi$.

(iv) Fill up $S^{\mathbf{3}} \times S^{\mathbf{1}} \times\{0\}$ by the Hopf circles in each $S^{\mathbf{3}}$. Q.E.D.

SMOOTHNESS. These curves are the orbits of the flow whose smoothness depends on the growth of curvature of $\gamma_{t}$. A class $C^{\infty}$ example is possible here. A very beautiful related example on $M^{3} \times S^{1} \times S^{1}$ due to Thurston is actually real analytic. $M^{3}$ is a nontrivial $S^{1}$ fibration over $S^{1} \times S^{1}$. A more detailed discussion of these examples will appear in [S].

Problems. Open problems are the questions of existence of such foliation examples (all leaves compact but volume unbounded) in codimension three or in the complex analytic context and finally for these new flows understanding the structure of infinities of the volume. See $[\mathbf{M}],[\mathbf{E}]$, and [EMS] for theorems about the nonexistence of this unbounded period phenomenon.

Note these pointwise periodic flows are "distal" in the sense of topological dynamics or "topologically rigid" in the sense of Zippin [Z].

\section{REFERENCES}

[M] D. Montgomery, Pointwise periodic homeomorphisms, Amer. J. Math. 59 (1937), 118-120.

[E] D. Epstein, Periodic flows on three-manifolds, Ann. of Math. (2) 95 (1972), 66-82. MR 44 \#5981.

[EMS] R. Edwards, K. Millet and D. Sullivan, Foliations with all leaves compact, Topology (submitted).

AMS (MOS) subject classifications (1970). Primary 58F99; Secondary 57D30, 54H20. 
[S] D. Sullivan, $A$ counterexample to the periodic orbit conjecture, Inst. Hautes Études Sci. Publ. Math. No. 46 (1976) (to appear).

[Z] L. Zippin, Transformation groups, Lectures in Topology, Univ. of Michigan Press, Ann. Arbor, Mich., 1941. MR 3, 134.

INSTITUT DES HAUTES ETUDES SCIENTIFIQUES, BURES-SUR-YVETTE, FR.ANCE 